\title{
Spaces of polynomial and nonpolynomial spline-wavelets
}

\author{
Yu. K. Dem'yanovich ${ }^{1, *}, I$. G. Burova ${ }^{1}, T$. O. Evdokimova ${ }^{1}$, and $A . V$. Lebedeva ${ }^{1}$ \\ ${ }^{1}$ St. Petersburg State University, 7/9 Universitetskaya nab., St.Petersburg, 199034 Russia
}

\begin{abstract}
This paper, discusses spaces of polynomial and nonpolynomial splines suitable for solving the Hermite interpolation problem (with first-order derivatives) and for constructing a wavelet decomposition. Such splines we call Hermitian type splines of the first level. The basis of these splines is obtained from the approximation relations under the condition connected with the minimum of multiplicity of covering every point of $(\alpha, \beta)$ (almost everywhere) with the support of the basis splines. Thus these splines belong to the class of minimal splines. Here we consider the processing of flows that include a stream of values of the derivative of an approximated function which is very important for good approximation. Also we construct a splash decomposition of the Hermitian type splines on a non-uniform grid.
\end{abstract}

\section{Introduction}

Recently, much attention has been paid to the application of wavelets to solving various problems. As it is known, the wavelet-Galerkin method is a useful tool for solving differential equations mainly because the conditional number of the stiffness matrix is independent of the matrix size and thus the number of iterations for solving the discrete problem by the conjugate gradient method is small. The authors of paper [1] have recently proposed a quadratic spline wavelet basis that has a small conditional number and short support. In this paper the authors used this basis in the Galerkin method to solve the second-order elliptic problems with Dirichlet boundary conditions in one and two dimensions and by an appropriate post-processing they achieve the $L_{2}$-error of order $O\left(h^{4}\right)$, where $\mathrm{h}$ is the step size. The rate of convergence is the same as the rate of convergence for the Galerkin method with cubic spline wavelets. They show theoretically, as well as numerically, that the presented method outperforms the Galerkin method with other quadratic or cubic spline wavelets. Furthermore, they present local post-processing for example of the equation with Dirac measure on the right-hand side.

In paper [2], to solve the problem of the high gradient adaptive analysis of the ship straight structure, a meshless local Petrov-Galerkin method based on a Bspline wavelet is proposed. The approximation function of the structural displacement field quantity is solved by employing the least squares method and the weighted residual method, and the governing equation and stiffness equation were established. Based on the meshless local Petrov-Galerkin method, an m-order Bspline function is used as the wavelet basis function to construct the approximation function of the ship structure displacement field, and a two-scale decomposition technology is used to decompose the high gradient component and the low scale component in the stress field. The high scale component is used to express the high gradient component in the stress field.

Composite materials, with characteristics of light weight and high strength, are useful in manufacturing. Therefore, precise design and analysis is the first key procedure in composite applications. Improper analysis or use of composite materials may cause serious failures. In paper [3], the wavelet finite element method (WFEM) based on B-spline wavelet on the interval (BSWI) is constructed for precise analysis of laminated plates and shells, which gives a guidance in design and application of composite structures. First, FEM formulations are derived from the generalized potential energy function based on the generalized variational principle and virtual work principle. Then, BSWI scaling functions are used as an interpolation function to discretize the solving displacement field variables. At the same time, a transformation matrix is constructed and used to translate the meaningless wavelet coefficients into physical space. At last, the static analysis results can be obtained by solving the FEM formulations.

The authors of paper [4] present a new biorthogonal wavelet transform using splines performed in a 'lifting' manner. Specifically, polynomial splines of different order were used in the lifting constructions. They study the influence of the order of filters using polynomial splines on image compression in order to choose the best wavelet transforms. In addition, a comparative study of these transforms is done firstly with the biorthogonal B9/7 transform which is frequently used in image compression and secondly with the existing B-spline based transforms. They show through experimental results that their proposed wavelet transforms outperform the existing ones in image compression.

In detailed aerodynamic design optimization, a large number of design variables in geometry parameterization are required to provide sufficient flexibility and obtain the potential optimum shape. However, with the increasing number of design variables, it becomes

* Corresponding author: yuri.demjanovich@gmail.com 
difficult to maintain the smoothness on the surface which consequently makes the optimization process progressively complex. In paper [5], smoothing methods based on B-spline functions are studied to improve the smoothness and design efficiency. The wavelet smoothing method and the least square smoothing method are developed through coordinate transformation in a linear space constructed by B-spline basis functions. In these two methods, smoothing is achieved by a mapping from the linear space to itself such that the design space remains unchanged. A design example is presented where aerodynamic optimization of a supercritical airfoil is conducted with smoothing methods included in the optimization loop. Affirmative results from the design example confirm that these two smoothing methods can greatly improve quality and efficiency compared with the existing conventional nonsmoothing method

In paper [6], Hermite wavelets are used to develop a numerical procedure for numerical solutions of twodimensional hyperbolic telegraph equation. In the first stage, the author rewrote the second order hyperbolic telegraph equation as a system of partial differential equations by introducing a new variable and then using finite difference approximation author discretized timedependent variables. After that, the Hermite wavelets series expansion is used for discretization of space variables. With this approach, finding the solution of a two-dimensional hyperbolic telegraph equation is transformed to finding the solution of two algebraic system of equations. The solution of these systems of algebraic equations gives Hermite wavelet coefficients. Then by inserting these coefficients into the Hermite wavelet series expansion, numerical solutions can be acquired consecutively. The main goal of this paper is to indicate that the Hermite wavelet-based method is suitable and efficient for a two-dimensional hyperbolic telegraph equation as well as other types of hyperbolic partial differential equations such as wave and sinhGordon equations. Six test problems are chosen and $L_{2}$, $L_{\infty}$ and root mean squared (RMS) error norms are measured for a comparison of current numerical results with exact results and with the results of previous studies based on such as meshless, B-spline and differential quadrature methods. The obtained results corroborate the applicability and efficiency of the proposed method.

To numerically solve the Burgers' equation, in this paper [7] the authors propose a general method for constructing wavelet bases on the interval $[0,1]$ derived from symmetric biorthogonal multiwavelets on the real line. In particular, they obtain wavelet bases with simple structures on the interval $[0,1]$ from the Hermite cubic splines. In comparison with all other known constructed wavelets on the interval $[0,1]$, authors constructed wavelet bases on the interval $[0,1]$ from the Hermite cubic splines not only have good approximation and symmetry properties with extremely short supports, but also employ a minimum number of boundary wavelets with a very simple structure. These desirable properties make them to be of particular interest in numerical algorithms. They constructed wavelet bases on the interval $[0,1]$ which are then used to solve the nonlinear
Burgers' equation. The method is based on the finite difference formula combined with the collocation method. Therefore, the proposed numerical scheme in this paper is abbreviated as MFDCM (Mixed Finite Difference and Collocation Method). Some numerical examples are provided to demonstrate the validity and applicability of the proposed method which can be easily implemented to produce a desired accuracy.

In [8] for cubic splines with nonuniform nodes, which split with respect to the even and odd nodes is used to obtain a wavelet expansion algorithm in the form of the solution to a three-diagonal system of linear algebraic equations for the coefficients. Computations by hand are used to investigate the application of this algorithm for numerical differentiation. The results are illustrated by solving a prediction problem.

The overview presented here shows the importance of taking into account the smoothness for the discussed functions and their derivatives. This paper, discusses spaces of polynomial and nonpolynomial splines suitable for solving the Hermite interpolation problem (with firstorder derivatives) and for constructing a wavelet decomposition. Such splines we call Hermitian type splines of the first level. The basis of these splines is obtained from the approximation relations under the condition connected with the minimum of multiplicity of covering every point of $(\alpha, \beta)$ (almost everywhere) with the support of the basis splines. Thus these splines belong to the class of minimal splines. This paper is ideologically similar to the papers [9], in which the spaces of the splines of the Lagrangian type are constructed.

Here we consider the processing of flows that include a stream of values of the derivative of an approximated function which is very important for good approximation. Also we construct a splash decomposition of the Hermitian type splines on a nonuniform grid.

The approximation and interpolation formulas for the stream under consideration are constructed. The obtained basis functions have compact support, and the addition of one node leads to an increase in the dimension of the spline space by two units (two basic wavelets are added to the previous basis). Sometimes to discuss the situation connected with a segment $[\mathrm{a}, \mathrm{b}] \subset(\alpha, \beta)$ is difficult. $\backslash$ Therefore we can solve this problem using our method by restricting all functions on the segment.

\section{Splines of Hermit Type}

Let $\varphi(t)=\left([\varphi]_{0}(t),[\varphi]_{1}(t),[\varphi]_{2}(t),[\varphi]_{3}(t)\right)^{T}$ be a four-component vector function with components $[\varphi]_{i}(t)$ from space $C^{1}(\alpha, \beta), \quad i=0,1,2,3$. Let condition (A) be fulfilled:

$$
\begin{gathered}
W(\mathrm{x}, \mathrm{y} ; \varphi) \stackrel{\text { def }}{=} \operatorname{det}\left(\varphi(x), \varphi^{\prime}(x), \varphi^{\prime}(y)\right) \neq 0 \\
\forall \mathrm{x}, \mathrm{y} \in(\alpha, \beta), \mathrm{x} \neq y .
\end{gathered}
$$

Let $\mathrm{X}$ be set of nodes such that 


$$
X: \alpha<\cdots<\mathrm{x}_{-1}<\mathrm{x}_{0}<\mathrm{x}_{1}<\cdots<\beta
$$

where $\alpha \stackrel{\text { def }}{=} \lim _{j \rightarrow-\infty} x_{j}, \beta \stackrel{\text { def }}{=} \lim _{j \rightarrow+\infty} x_{j}$.

Let us denote

$$
\mathrm{G} \stackrel{\text { def }}{=} \cup_{j \in \mathbb{Z}}\left(x_{j}, \mathrm{X}_{\mathrm{j}+1}\right), \varphi_{j} \stackrel{\text { def }}{=} \varphi\left(x_{j}\right), \varphi^{\prime}{ }_{j} \stackrel{\text { def }}{=} \varphi^{\prime}\left(x_{j}\right) .
$$

Suppose functions $\omega_{j}(t), \mathrm{t} \in G, \mathrm{j} \in \mathbb{Z}$, are the solution of the system of equations, which we call the approximation relations

$$
\sum\left(\varphi_{\mathrm{j}+1}^{\prime} \omega_{2 j-1}(t)+\varphi_{\mathrm{j}+1} \omega_{2 j}(t)\right)=\varphi(t) .
$$

Here we suppose that

$$
\begin{gathered}
\operatorname{supp} \omega_{2 j-1} \subset\left[x_{j}, x_{j+2}\right], \\
\operatorname{supp}_{2 j} \subset\left[x_{j}, x_{j+2}\right] \forall j \in \mathbb{Z} .
\end{gathered}
$$

We obtain from (2.2) - (2.3) for $t \in\left(x_{k}, \mathrm{x}_{\mathrm{k}+1}\right)$ with fixed $k \in \mathbb{Z}$

$$
\begin{gathered}
\varphi_{k}^{\prime} \omega_{2 k-3}(t)+\varphi_{k} \omega_{2 k-2}(t)+ \\
+\varphi_{\mathrm{k}+1}^{\prime} \omega_{2 k-1}(t)+\varphi_{\mathrm{k}+1} \omega_{2 k}(t)=\varphi(t) .
\end{gathered}
$$

Due to property (A), the solution of system (2.4) is unique. Let $t \in\left(x_{k}, \mathrm{x}_{\mathrm{k}+1}\right)$. In this case the solution of the system can be written in the form:

$$
\begin{gathered}
\omega_{2 k-3}(t)=\frac{\operatorname{det}\left(\varphi(t), \varphi_{\mathrm{k}^{\prime}}, \varphi_{\mathrm{k}+1}^{\prime}, \varphi_{\mathrm{k}+1}\right)}{\operatorname{det}\left(\varphi_{k_{k}}^{\prime}, \varphi_{k}, \varphi_{\mathrm{k}+1}^{\prime}, \varphi_{\mathrm{k}+1}\right)}, \\
\omega_{2 k-2}(t)=\frac{\operatorname{det}\left(\varphi_{k}^{\prime} \varphi(t), \varphi_{\mathrm{k}+1}^{\prime}, \varphi_{\mathrm{k}+1}\right)}{\operatorname{det}\left(\varphi_{k^{\prime}}^{\prime}, \varphi_{k}, \varphi_{\mathrm{k}+1}^{\prime}, \varphi_{\mathrm{k}+1}\right)}, \\
\omega_{2 k-1}(t)=\frac{\operatorname{det}\left(\varphi_{k^{\prime}}, \varphi_{k}, \varphi(t), \varphi_{\mathrm{k}+1}\right)}{\operatorname{det}\left(\varphi_{{ }_{k}}^{\prime}, \varphi_{k}, \varphi_{\mathrm{k}+1}^{\prime}, \varphi_{\mathrm{k}+1}\right)} \\
\omega_{2 k}(t)=\frac{\operatorname{det}\left(\varphi_{k^{\prime}}^{\prime}, \varphi_{k}, \varphi_{\mathrm{k}+1}^{\prime}, \varphi(t)\right)}{\operatorname{det}\left(\varphi_{{ }_{k}}^{\prime}, \varphi_{k}, \varphi_{\mathrm{k}+1}^{\prime}, \varphi_{\mathrm{k}+1}\right)}
\end{gathered}
$$

Now if $k=q, k=q+1$, we get for any $q \in \mathbb{Z}$ :

$$
\omega_{2 q-1}(t)=\frac{\operatorname{det}\left(\varphi_{q}^{\prime}, \varphi_{q}, \varphi(t), \varphi_{\mathrm{q}+1}\right)}{\operatorname{det}\left(\varphi_{q}^{\prime}, \varphi_{q}, \varphi_{\mathrm{q}+1}^{\prime}, \varphi_{\mathrm{q}+1}\right)^{\prime}}
$$

where $t \in\left(x_{q}, \mathrm{x}_{\mathrm{q}+1}\right)$,

$$
\omega_{2 q-1}(t)=\frac{\operatorname{det}\left(\varphi(t), \varphi_{\mathrm{q}+1}, \varphi_{\mathrm{q}+2}^{\prime}, \varphi_{\mathrm{q}+2}\right)}{\operatorname{det}\left(\varphi_{\mathrm{q}+1}^{\prime}, \varphi_{\mathrm{q}+1}, \varphi_{\mathrm{q}+2}^{\prime}, \varphi_{\mathrm{q}+2}\right)},
$$

where $t \in\left(x_{\mathrm{q}+1}, \mathrm{x}_{\mathrm{q}+2}\right)$,

$$
\omega_{2 q}(t)=\frac{\operatorname{det}\left(\varphi_{q}^{\prime}, \varphi_{q}, \varphi_{\mathrm{q}+1}^{\prime}, \varphi(t)\right)}{\operatorname{det}\left(\varphi_{q}^{\prime}, \varphi_{q}, \varphi_{\mathrm{q}+1}^{\prime}, \varphi_{\mathrm{q}+1}\right)},
$$

where $t \in\left(x_{q}, \mathrm{x}_{\mathrm{q}+1}\right)$,

$$
\omega_{2 q}(t)=\frac{\operatorname{det}\left(\varphi_{\mathrm{q}+1}^{\prime}, \varphi(t), \varphi_{\mathrm{q}+2}^{\prime}, \varphi_{\mathrm{q}+2}\right)}{\operatorname{det}\left(\varphi_{\mathrm{q}+1}^{\prime}, \varphi_{\mathrm{q}+1}, \varphi_{\mathrm{q}+2}^{\prime}, \varphi_{\mathrm{q}+2}\right)},
$$

where $t \in\left(x_{\mathrm{q}+1}, \mathrm{x}_{\mathrm{q}+2}\right)$.

Theorem 1. Let $\varphi \in C^{1}(\alpha, \beta)$ and let condition (A) be fulfilled; then for any $q \in Z$ functions $\omega_{2 q-1}(t)$ и $\omega_{2 q}(t)$, which are given by (2.3) и (2.5) - (2.8), can be continued by continuity for the entire interval $(\alpha, \beta)$ to class functions $C^{1}(\alpha, \beta)$. In addition, the following relations are valid:

$\omega_{2 q-1}\left(x_{q}\right)=0, \omega_{2 q-1}\left(x_{\mathrm{q}+1}\right)=$
$0, \omega_{2 q-1}\left(x_{\mathrm{q}+2}\right)=0$,

$\omega_{2 \mathrm{q}-1}^{\prime}\left(x_{q}\right)=0, \omega_{2 \mathrm{q}-1}^{\prime}\left(x_{\mathrm{q}+1}\right)=$

$1, \omega_{2 \mathrm{q}-1}^{\prime}\left(x_{\mathrm{q}+2}\right)=0$,

0 ,

$$
\omega_{2 q}\left(x_{q}\right)=0, \omega_{2 q}\left(x_{\mathrm{q}+1}\right)=1, \omega_{2 q}\left(x_{\mathrm{q}+2}\right)=
$$

$$
\omega_{2 q}\left(x_{q}\right)=0, \omega_{2 q}\left(x_{\mathrm{q}+1}\right)=1, \omega_{2 q}\left(x_{\mathrm{q}+2}\right)=
$$

0 ,

where previous designations are used for continued functions.

Proof. Calculating the corresponding one-sided limits from the functions $\omega_{2 q-1}(t)$ and $\omega_{2 q}(t)$ and their derivatives at nodes $x_{q}, x_{q+1}$ and $x_{q+2}$ with the help of representations (2.3) and (2.5) - (2.8), we conclude that all the statements in the theorem are valid (see also [9]).

Remark 1. If the components $[\varphi(t)]_{i}$ of the vector $\varphi(t)$ are given by the equations $[\varphi(t)]_{i}=\mathrm{t}^{i}$, then the functions $\omega_{2 q-1}(t)$ and $\omega_{2 q}(t)$ represent the known interpolation basis of the cubic Hermitian spline space.

The space

$$
S_{\varphi}^{1}(X) \stackrel{\text { def }}{=}\left\{u \mid \mathrm{u}=\sum c_{j} \omega_{j} \forall c_{j} \in \mathbb{R}^{1, j} \in \mathbb{Z}\right\}
$$

is called the spline space of Hermitian type (of the first level). In view of the property (A), the functions $\omega_{j}$, $j \in \mathbb{Z}$, are linearly independent. The set $\omega_{j}, j \in \mathbb{Z}$, is called the main basis of the space $S_{\varphi}^{1}(X)$.

Remark 2. Relations (2.9) - (2.12) can be written in the form

$$
\begin{gathered}
\omega_{2 s-1}\left(x_{j}\right)=0, \omega_{2 s-1}^{\prime}\left(x_{j}\right)=\delta_{s+1, j}, \\
\omega_{2 s}\left(x_{j}\right)=\delta_{s+1, j}, \omega_{2 s}^{\prime}\left(x_{j}\right)=0 \forall s, j \in \mathbb{Z} .
\end{gathered}
$$




\section{Calibration relations for the Hermitian type splines}

In the set $X$, consider the subset $Y$ :

$$
\begin{gathered}
Y: \ldots<y_{-2}<y_{-1}<y_{0}<y_{1}<y_{2}<\ldots \\
\lim y_{j}=\alpha, \lim y_{j}=\beta .
\end{gathered}
$$

Let $\chi(s)$ denote a monotonically increasing integer function such that

$$
y_{j}=x_{\chi}(j)
$$

Let $\mathbb{Z}^{\chi}=\chi(\mathbb{Z})$. The introduced function is reversible on $\mathbb{Z}^{\chi}$ and generates the map $Y \mapsto X$ which is the embedding of $Y$ in $X$. Repeating constructions (2.2) (2.8) using the newly introduced grid $Y$, the functions $w_{j}$, for which

$$
\begin{gathered}
\operatorname{supp} w_{2 j-1} \subset\left[y_{j}, \mathrm{y}_{\mathrm{j}+2}\right], \\
\operatorname{supp} w_{2 j} \subset\left[y_{j}, \mathrm{y}_{\mathrm{j}+2}\right] \forall j \in \mathbb{Z} .
\end{gathered}
$$

For a fixed $i \in Z$ for $t \in\left(y_{i}, \mathrm{y}_{\mathrm{i}+1}\right)$, by analogy with (2.4) we have

$$
\begin{gathered}
\mathrm{v}_{i}^{\prime} w_{2 i-3}(t)+\mathrm{v}_{i} w_{2 i-2}(t)+ \\
+\mathrm{v}_{\mathrm{i}+1}^{\prime} w_{2 i-1}(t)+\mathrm{v}_{\mathrm{i}+1} w_{2 i}(t)=\varphi(t),
\end{gathered}
$$

where $v_{j}=\varphi_{y_{j}}, \mathrm{v}_{j}^{\prime}=\varphi_{y_{j}}^{\prime}, \forall j \in \mathbb{Z}$.

We find from relations (3.2) - (3.3) for $p \in \mathbb{Z}$

$$
w_{2 p-1}(t)=\frac{\operatorname{det}\left(v_{p}^{\prime}, v_{p}, \varphi(t), v_{p+1}\right)}{\operatorname{det}\left(v_{p}^{\prime}, v_{p}, v_{p+1}^{\prime}, v_{p+1}\right)},
$$

where $t \in\left(y_{p}, y_{p+1}\right)$,

$$
w_{2 p-1}(t)=\frac{\operatorname{det}\left(\varphi(t), v_{p+1}, v_{p+2}^{\prime}, v_{p+2}\right)}{\operatorname{det}\left(v_{p+1}^{\prime}, v_{p+1}, v_{p+2}^{\prime}, v_{p+2}\right)}
$$

where $t \in\left(y_{p+1}, y_{p+2}\right)$,

$$
w_{2 p}(t)=\frac{\operatorname{det}\left(v_{p}^{\prime}, v_{p}, v_{p+1}^{\prime}, \varphi(t)\right)}{\operatorname{det}\left(v_{p}^{\prime}, v_{p}, v_{p+1}^{\prime}, v_{p+1}\right)}
$$

where $t \in\left(y_{p}, y_{p+1}\right)$,

$$
w_{2 p}(t)=\frac{\operatorname{det}\left(v_{p+1}^{\prime}, \varphi(t), v_{p+2}^{\prime}, v_{p+2}\right)}{\operatorname{det}\left(v_{p+1}^{\prime}, v_{p+1}, v_{p+2}^{\prime}, v_{p+2}\right)}
$$

where $t \in\left(y_{p+1}, y_{p+2}\right)$.

It can be shown that for functions (3.4) - (3.7) the following equations are valid, they are similar to (2.13) (2.14)

$$
\begin{gathered}
w_{2 s-1}\left(y_{j}\right)=0, w_{2 s-1}^{\prime}\left(y_{j}\right)=\delta_{s+1, j}, \\
w_{2 s}\left(y_{j}\right)=\delta_{s+1, j}, w^{\prime}{ }_{2 s}\left(y_{j}\right)=0 \forall s, j \in \mathbb{Z}
\end{gathered}
$$

Let $q=\chi(i), q+k=\chi(i+1)$, so that between nodes $y_{i}$ and $y_{i+1}$ there are nodes $x_{j}, j=q+1, q+2, \ldots, q+k-1$ :

$$
\begin{aligned}
& y_{i}=x_{q}<x_{q+1}<x_{q+2}<\ldots<x_{q+k-1}< \\
& x_{q+k}=y_{i+1} .
\end{aligned}
$$

It was shown in [9] that if one node is removed from the original grid, the coordinate functions $w_{j}$ associated with the new grid are linear combinations of the original (the mentioned linear combinations are called gauge relations). It follows that when a group of nodes is deleted, the corresponding coordinate functions will also have this property. To determine the coefficients of the gauge relations, we use the biorthogonal system of functionals represented by formulas (3.8) - (3.9). Thus, taking into account the arrangement of the supports of the functions $w_{j}, j \in\{2 i-3,2 i-2,2 i-1,2 i\}$ and the functions $\omega_{2 s-3}, \omega_{2 s-2}, \omega_{2 s-1}, \omega_{2 s}$, (see formulas (2.3)), for $t \in\left(y_{i}, y_{i+1}\right)$ and we have the representations

$w_{j}(t)=$

$\sum_{\left(y_{i}, y_{i+1}\right) \cap\left(x_{s}, x_{s+2}\right) \neq \emptyset}\left(c_{2 s-1}^{(j)} \omega_{2 s-1}(t)+c_{2 s}^{(j)} \omega_{2 s}(t)\right)$,

where $j \in\{2 i-3,2 i-2,2 i-1,2 i\}$.

The following statement is true.

Theorem 1. Let $i$ be a fixed integer and $k=\chi(i+1)$ $\chi(i)+1$. Under these conditions, when $t \in\left(y_{i}, y_{i+1}\right)$, the following relations are true

$$
\begin{gathered}
w_{j}(t)= \\
=\sum_{s=q-1}^{q+k-1}\left(w_{j}^{\prime}\left(x_{s+1}\right) \omega_{2 s-1}(t)+w_{j}\left(x_{s+1}\right) \omega_{2 s}(t)\right),
\end{gathered}
$$

where $j \in\{2 i-3,2 i-2,2 i-1,2 i\}, q=\chi(i)$.

Proof. Relation (3.11) can be rewritten as

$w_{j}(t)=\sum_{s=q-1}^{q+k-1}\left(c_{2 s-1}^{(j)} \omega_{2 s-1}(t)+c_{2 s}^{(j)} \omega_{2 s}(t)\right)$,

where $j \in\{2 i-3,2 i-2,2 i-1,2 i\}$.

Substituting $t=x_{r}, r \in\{\mathrm{q}, \mathrm{q}+1, \ldots, \mathrm{q}+\mathrm{k}\}$ into formula (3.13), we have

$w_{j}\left(x_{r}\right)=\sum_{s=q-1}^{q+k-1}\left(c_{2 s-1}^{(j)} \omega_{2 s-1}\left(x_{r}\right)+c_{2 s}^{(j)} \omega_{2 s}\left(x_{r}\right)\right)$.

Using the relations

$$
\omega_{2 s-1}\left(x_{r}\right)=0, \omega_{2 s}\left(x_{r}\right)=\delta_{s+1, r},
$$

we find only one nonzero term on the right-hand side of (3.14); thus, we find a term with the index $s=r-1$ :

$$
w_{j}\left(x_{r}\right)=c_{2 r-2}^{(j)} \omega_{2 r-2}\left(x_{r}\right)=c_{2 r-2}^{(j)} .
$$

So,

$$
c_{2 s}^{(j)}=w_{j}\left(x_{s+1}\right) \forall s \in\{q-1, q, \ldots, q+k-1\} .
$$


Differentiating relation (3.15) and substituting $t=x_{r}$ into the obtained identity, we find

$$
\begin{aligned}
& w_{j}^{\prime}\left(x_{r}\right)=\sum_{s=q-1}^{q+k-1}\left(c_{2 s-1}^{(j)} \omega_{2 s-1}^{\prime}\left(x_{r}\right)+\right. \\
& \left.c_{2 s}^{(j)} \omega_{2 s}^{\prime}\left(x_{r}\right)\right) .
\end{aligned}
$$

Considering the relations $\omega_{2 \mathrm{~s}-1}^{\prime}\left(x_{r}\right)=\delta_{\mathrm{s}+1, \mathrm{r}}$, $\omega_{2 s}\left(x_{r}\right)=0$, we see that on the right side of the equation (3.16) there is perhaps only one nonzero term (in this case, it is the first one), thus, we find a term with the subscript $s=r-1$; Thus, we have $w_{j}^{\prime}\left(x_{r}\right)=$ $c_{2 r-3}^{(j)}$ and

$c_{2 s-1}^{(j)}=w_{j}^{\prime}\left(x_{s+1}\right) \forall s \in\{q-1, q, \ldots, q+k-1\}$.

Substituting (3.15) and (3.17) into (3.13), we find relations (3.12).

Theorem 2. Under the conditions of Theorem 1, relations (3.12) can be represented in the form

$$
\begin{gathered}
w_{2 i-3}(t)=\omega_{2 q-3}(t)+ \\
+\sum_{s^{\prime}=q+1}^{q+k-1} \quad w_{2 i-3}^{\prime}\left(x_{s^{\prime}}\right) \omega_{2 s^{\prime}-3}(t)+ \\
+\sum_{s^{\prime}=q+1}^{q+k-1} \quad w_{2 i-3}\left(x_{s^{\prime}}\right) \omega_{2 s^{\prime}-2}(t) . \\
w_{2 i-2}(t)=\omega_{2 q-2}(t)+ \\
+\sum_{s^{\prime}=q+1}^{q+k-1} \quad w_{2 i-2}^{\prime}\left(x_{s^{\prime}}\right) \omega_{2 s^{\prime}-3}(t)+ \\
+\sum_{s^{\prime}=q+1}^{q+k-1} \quad w_{2 i-2}\left(x_{s^{\prime}}\right) \omega_{2 s^{\prime}-2}(t) .
\end{gathered}
$$

$w_{2 i-1}(t)=\sum_{s^{\prime}=q+1}^{q+k-1} \quad w^{\prime}{ }_{2 i-1}\left(x_{s^{\prime}}\right) \omega_{2 s^{\prime}-3}(t)+$

$+\sum_{s^{\prime}=q+1}^{q+k-1} \quad w_{2 i-1}\left(x_{s^{\prime}}\right) \omega_{2 s^{\prime}-1}(t)+\omega_{2 q+2 k-3}(t)$.

$$
\begin{gathered}
w_{2 i}(t)=\sum_{s^{\prime}=q+1}^{q+k-1} \quad w_{2 i}^{\prime}\left(x_{s^{\prime}}\right) \omega_{2 s^{\prime}-3}(t)+ \\
+\sum_{s^{\prime}=q+1}^{q+k-1} \quad w_{2 i}\left(x_{s^{\prime}}\right) \omega_{2 s^{\prime}-2}(t)+\omega_{2 q+2 k-2}(t) .
\end{gathered}
$$

Proof. Formula (3.12) can be represented as

$$
\begin{aligned}
& \quad w_{j}(t)=w_{j}^{\prime}\left(x_{q}\right) \omega_{2 q-3}(t)+w_{j}\left(x_{q}\right) \omega_{2 q-2}(t)+ \\
& +\sum_{s=q}^{q+k-2}\left(w_{j}^{\prime}\left(x_{s+1}\right) \omega_{2 s-1}(t)+w_{j}\left(x_{s+1}\right) \omega_{2 s}(t)\right)+ \\
& +w_{j}^{\prime}\left(x_{q+k}\right) \omega_{2 q+2 k-3}(t)+w_{j}\left(x_{q+k}\right) \omega_{2 q+2 k-2}(t) ; \\
& \text { considering that } x_{q}=y_{i} \text { and } x_{q+k}=y_{i+1} \text { we get }
\end{aligned}
$$

$$
\begin{gathered}
w_{j}(t)=w_{j}^{\prime}\left(y_{i}\right) \omega_{2 q-3}(t)+w_{j}\left(y_{i}\right) \omega_{2 q-2}(t)+ \\
+\sum_{s=q}^{q+k-2}\left(w_{j}^{\prime}\left(x_{s+1}\right) \omega_{2 s-1}(t)+w_{j}\left(x_{s+1}\right) \omega_{2 s}(t)\right)+ \\
+w_{j}^{\prime}\left(y_{i+1}\right) \omega_{2 q+2 k-3}(t)+w_{j}\left(y_{i+1}\right) \omega_{2 q+2 k-2}(t) .
\end{gathered}
$$

From formula (3.22) with $j=2 i-3$, we have

$$
\begin{gathered}
w_{2 i-3}(t)=w_{2 i-3}^{\prime}\left(y_{i}\right) \omega_{2 q-3}(t)+ \\
+w_{2 i-3}\left(y_{i}\right) \omega_{2 q-2}(t)+ \\
+\sum_{S=q}^{q+k-2} \quad w^{\prime}{ }_{2 i-3}\left(x_{s+1}\right) \omega_{2 s-1}(t)+ \\
+\sum_{S=q}^{q+k-2} \quad w_{2 i-3}\left(x_{s+1}\right) \omega_{2 s}(t)+ \\
+w_{2 i-3}^{\prime}\left(y_{i+1}\right) \omega_{2 q+2 k-3}(t)+ \\
+w_{2 i-3}\left(y_{i+1}\right) \omega_{2 q+2 k-2}(t)
\end{gathered}
$$

From formulas (3.8) - (3.9) we get:

$$
\begin{gathered}
w_{2 i-3}^{\prime}\left(y_{i}\right)=1 \\
w_{2 i-3}\left(y_{i}\right)=w_{2 i-3}^{\prime}\left(y_{i+1}\right)=w_{2 i-3}\left(y_{i+1}\right)=0,
\end{gathered}
$$

and therefore identity (3.23)can be given the form (3.18). When $j=2 i-2$, the identity (3.22) takes the form

$$
\begin{gathered}
w_{2 i-2}(t)=w_{2 i-2}^{\prime}\left(y_{i}\right) \omega_{2 q-3}(t)+ \\
+w_{2 i-2}\left(y_{i}\right) \omega_{2 q-2}(t)+ \\
+\sum_{s=q}^{q+k-2} \quad w^{\prime}{ }_{2 i-2}\left(x_{s+1}\right) \omega_{2 s-1}(t)+ \\
+\sum_{s=q}^{q+k-2} \quad w_{2 i-2}\left(x_{s+1}\right) \omega_{2 s}(t)+ \\
+w^{\prime}{ }_{2 i-2}\left(y_{i+1}\right) \omega_{2 q+2 k-3}(t)+ \\
+w_{2 i-2}\left(y_{i+1}\right) \omega_{q+2 k-2}(t) .
\end{gathered}
$$

using equalities (3.8) - (3.9), we find

$$
\begin{gathered}
w_{2 i-2}^{\prime}\left(y_{i}\right)=0, w_{2 i-2}\left(y_{i}\right)=1, \\
w_{2 i-2}^{\prime}\left(y_{i+1}\right)=w_{2 i-2}\left(y_{i+1}\right)=0,
\end{gathered}
$$

so from (3.24) we derive the formula (3.19). Consider (3.2) with $j=2 i-1$ :

$$
\begin{gathered}
w_{2 i-1}(t)=w_{2 i-1}^{\prime}\left(y_{i}\right) \omega_{2 q-3}(t)+ \\
+w_{2 i-1}\left(y_{i}\right) \omega_{2 q-2}(t)+ \\
+\sum_{s=q}^{q+k-2} \quad w_{2 i-1}^{\prime}\left(x_{s+1}\right) \omega_{2 s-1}(t)+ \\
+\sum_{S=q}^{q+k-2} w_{2 i-1}\left(x_{s+1}\right) \omega_{2 s}(t)+ \\
+w_{2 i-1}^{\prime}\left(y_{i+1}\right) \omega_{2 q+2 k-3}(t)+ \\
+w_{2 i-1}\left(y_{i+1}\right) \omega_{2 q+2 k-2}(t) .
\end{gathered}
$$

Using (3.8) - (3.9), we have

$$
w^{\prime}{ }_{2 i}\left(y_{i}\right)=w_{2 i}\left(y_{i}\right)=w_{2 i}^{\prime}\left(y_{i+1}\right)=0,
$$




$$
w_{2 i-1}\left(y_{i+1}\right)=0,
$$

from (3.25) we find the relation (3.20). Finally, consider the case $j=2 i$; in this case (3.22) takes the form:

$$
\begin{gathered}
w_{2 i}(t)=w_{2 i}^{\prime}\left(y_{i}\right) \omega_{2 q-3}(t)+ \\
+w_{2 i}\left(y_{i}\right) \omega_{2 q-2}(t)+ \\
+\sum_{s=q}^{q+k-2} \quad w^{\prime}{ }_{2 i}\left(x_{s+1}\right) \omega_{2 s-1}(t)+ \\
+\sum_{s=q}^{q+k-2} \quad w_{2 i}\left(x_{s+1}\right) \omega_{2 s}(t)+ \\
+w_{2 i}^{\prime}\left(y_{i+1}\right) \omega_{2 q+2 k-3}(t)+ \\
+w_{2 i}\left(y_{i+1}\right) \omega_{2 q+2 k-2}(t) .
\end{gathered}
$$

From (3.8) - (3.9) we get

$$
\begin{gathered}
w_{2 i}^{\prime}\left(y_{i}\right)=w_{2 i}\left(y_{i}\right)=w_{2 i}^{\prime}\left(y_{i+1}\right)=0, \\
w_{2 i}\left(y_{i+1}\right)=1,
\end{gathered}
$$

and therefore (3.25) can be represented in the form (3.21).

The theorem is proved.

Corollary 1. If the conditions of Theorem 1 and, $k=2$ are satisfied, then the relations can be given the form

$$
\begin{gathered}
w_{2 i-3}(t)=\omega_{2 q-2}(t)+\mathrm{w}_{2 i-3}^{\prime}\left(x_{\mathrm{q}+1}\right) \omega_{2 q-1}(t)+ \\
+\mathrm{w}_{2 i-3}\left(x_{\mathrm{q}+1}\right) \omega_{2 q}(t) . \\
w_{2 i-2}(t)=\omega_{2 q-2}(t)+\mathrm{w}_{2 i-2}^{\prime}\left(x_{\mathrm{q}+1}\right) \omega_{2 q-1}(t)+ \\
\quad+\mathrm{w}_{2 i-2}\left(x_{\mathrm{q}+1}\right) \omega_{2 q}(t), \\
w_{2 i-1}(t)=\mathrm{w}_{2 i-1}^{\prime}\left(x_{\mathrm{q}+1}\right) \omega_{2 q-1}(t) \\
+\mathrm{w}_{2 i-1}\left(x_{\mathrm{q}+1}\right) \omega_{2 q}(t)+\omega_{2 \mathrm{q}+1}(t), \\
\quad w_{2 i}(t)=\mathrm{w}_{2 i}^{\prime}\left(x_{\mathrm{q}+1}\right) \omega_{2 q-1}(t) \\
+
\end{gathered}
$$

Proof. Putting $k=2$ in relations (3.18), (3.19), (3.20), (3.21) we obtain the identities (3.27), (3.28), (3.29), (3.30), respectively.

Remark 3. In an algorithmic implementation, it is useful to remember that the case $k=1$ corresponds to mapping $\chi$, in which there are no nodes of grid $\mathrm{X}$ between the nodes $y_{i}$ and $y_{i+1}$, i.e. $\chi(i)=q, \chi(i+1)=q+1$, so that $y_{i}=x_{q}, \quad y_{i+1}=x_{q+1} \quad$ (see (3.1) and (3.10)); Moreover, if we assume that for $m>n$, expression $\sum_{j=m}^{n} \quad a_{j}$ equals zero (by definition) then the formulas of Theorems 1 and 2 are also valid in the case of $k=1$.

Now we assume that $q=\chi(i), q+k=\chi(i+1), q$ $k^{\prime}=\chi(i-1)$, so that there are nodes $x_{j}, j=q-1, q-2, \ldots$, $q-k+1$, between nodes $y_{i-1}$ and $y_{i}$, and there are nodes $x_{j}, j=q+1, q+2, \ldots, q+k-1$, between nodes $\mathrm{y}_{\mathrm{i}}$ and $y_{i+1}$ :

$$
\begin{aligned}
& y_{i-1}=\mathrm{x}_{q-k^{\prime}}<\mathrm{x}_{q-k^{\prime}+1}<\ldots<\mathrm{x}_{q-2}<\mathrm{x}_{q-1}<\mathrm{y}_{i}= \\
& =\mathrm{x}_{q}<\mathrm{x}_{\mathrm{q}+1}<x_{q+2}<\ldots<x_{q+k-1}<x_{q+k}=y_{i+1}
\end{aligned}
$$

Theorem 3. If condition (A) is satisfied, and $t \in(\alpha, \beta)$ then for any $i \in \mathbb{Z}$ the following relations are valid:

$w_{j}(t)=$

$\sum_{\mathrm{s}=\mathrm{q}^{\prime}}^{\mathrm{q}+\mathrm{k}-2}\left(\mathrm{w}_{j}^{\prime}\left(x_{\mathrm{s}+1}\right) \omega_{2 s-1}(t)+\mathrm{w}_{j}\left(x_{\mathrm{s}+1}\right) \omega_{2 s}(t)\right)$,

where $j \in\{2 i-3,2 i-2\}, \quad q=\chi(i), \quad q^{\prime}=\chi(i-1)$, $k=\chi(i+1)$.

Proof. The support of the functions $w_{j}, j=2 i-3,2 i$ 2 is located on the segment $\left[y_{i-1}, y_{i+1}\right]$. When $t \in$ $\left(y_{i}, \mathrm{y}_{\mathrm{i}+1}\right)$ formula (3.32) is valid according to Theorem 1. Consider the interval $t \in\left(y_{i-1}, \mathrm{y}_{i}\right)$. Replacing in Theorem $1 i$ by $i-1, q$ by $q^{\prime}$, and $k$ by $\mathrm{k}^{\prime} \stackrel{\text { def }}{=} \chi(i)-$ $\chi(i-1)$, we have

$$
\begin{gathered}
w_{j}(t)= \\
=\sum_{\mathrm{s}^{\prime}=\mathrm{q}^{\prime}-1}^{\mathrm{q}^{\prime}-1}\left(\mathrm{w}_{j}^{\prime}\left(x_{\mathrm{s}^{\prime}+1}\right) \omega_{2 \mathrm{~s}^{\prime}-1}(t)+\mathrm{w}_{j}\left(x_{\mathrm{s}^{\prime}+1}\right) \omega_{2 \mathrm{~s}^{\prime}}(t)\right) .
\end{gathered}
$$

Note that, according to the notation (3.31), the nodes $x_{q^{\prime}+\mathrm{k}^{\prime}}$ and $x_{k}$ coincide with the node $y_{i}$, and $q^{\prime}+\mathrm{k}^{\prime}=\mathrm{k}$. Therefore, in the sum (3.33), the term corresponding to the index $s^{\prime}=\mathrm{q}^{\prime}+\mathrm{k}^{\prime}-1$ coincides with the term in the sum (3.12) calculated for the index $s=q-1$. There are no other common terms in these sums. Considering this circumstance and the fact that at the ends of the interval $\left[y_{i}, \mathrm{y}_{\mathrm{i}+1}\right]$ the corresponding terms are equal to zero, we conclude that the union of the sums (3.12) and (3.33) leads to the formula (3.32).

Remark 4. Introducing the replacement index $i^{\prime}=\mathrm{i}-1$, we get

$$
\mathrm{q}=\mathrm{\chi}\left(\mathrm{i}^{\prime}+1\right), \mathrm{q}^{\prime}=\mathrm{\chi}\left(\mathrm{i}^{\prime}\right), \mathrm{k}=\mathrm{\chi}\left(\mathrm{i}^{\prime}+2\right)-q \text {. }
$$

If we put $S^{\prime}=\mathrm{s}+1$, then the formula (3.32) can be written in the following equivalent form

$$
\begin{gathered}
w_{j}(t)= \\
=\sum_{\mathrm{s}^{\prime}=\mathrm{q}^{\prime}+1}^{\mathrm{q}^{+}-1}\left(\mathrm{w}_{j}^{\prime}\left(x_{\mathrm{s}^{\prime}}\right) \omega_{2 \mathrm{~s}^{\prime}-3}(t)+\mathrm{w}_{j}\left(x_{\mathrm{s}^{\prime}}\right) \omega_{2 \mathrm{~s}^{\prime}-2}(t)\right), \\
j \in\left\{2 i^{\prime}-1,2 i^{\prime}\right\}, i^{\prime} \in \mathbb{Z} .
\end{gathered}
$$

For each $i \in \mathbb{Z}$ we consider $j \in\{2 i-1,2 i\}$ and consider

$$
\mathrm{q}=\chi(\mathrm{i}+1), \mathrm{q}^{\prime}=\chi(i), \mathrm{k}=\chi(\mathrm{i}+2)-q .
$$


Using (3.35) we have

$$
\begin{gathered}
w_{j}(t)= \\
=\sum_{s=\chi(i)}^{\chi(\mathrm{i}+2)}\left(\mathrm{w}_{j}^{\prime}\left(x_{s}\right) \omega_{2 s-3}(t)+\mathrm{w}_{j}\left(x_{s}\right) \omega_{2 s-2}(t)\right) .
\end{gathered}
$$

Since it is obvious that $\mathrm{w}_{j}^{\prime}\left(x_{\chi(i)}\right)=\mathrm{w}_{j}^{\prime}\left(x_{\chi(\mathrm{i}+2)}\right)=0$ and $w_{j}\left(x_{\chi(i)}\right)=\mathrm{w}_{j}\left(x_{\chi(\mathrm{i}+2)}\right)=0$, so the previous relation can be written as

$$
\begin{gathered}
w_{j}(t)= \\
=\sum_{\mathrm{s}=\chi(i)+1}^{\chi(\mathrm{i}+2)-1}\left(\mathrm{w}_{j}^{\prime}\left(x_{s}\right) \omega_{2 s-3}(t)+\mathrm{w}_{j}\left(x_{s}\right) \omega_{2 s-2}(t)\right) .
\end{gathered}
$$

For each $i \in \mathbb{Z}, j \in\{2 i-1,2 i\}$ consider the numbers $p_{j, k}$ for every $k \in \mathbb{Z}$ determined by the relations

$$
\begin{aligned}
& p_{j, 2 \sigma-3}=\mathrm{w}_{j}^{\prime}\left(x_{\sigma}\right), \mathrm{p}_{j, 2 \sigma-2}=\mathrm{w}_{j}\left(x_{\sigma}\right) \\
& \forall \sigma \in\{\chi(i)+1, \ldots, \chi(\mathrm{i}+2)-1\},
\end{aligned}
$$

and the numbers not mentioned in this list numbers $p_{j, k}$ will be considered as equal to zero:

$$
\begin{gathered}
p_{j, k}=0 \quad \forall j \in \mathbb{Z} \\
\forall k \notin\{2 \chi(i)-1,2 \chi(i), \ldots, 2 \chi(\mathrm{i}+2)-4\} .
\end{gathered}
$$

We denote $\boldsymbol{P}$ an infinite matrix, $P=()_{j, k \in \mathbb{Z}}$, whose elements are given by equations (3.38) - (3.39).

Thus, the row of the matrix $\boldsymbol{P}$ with the number $2 i-1$ is

$$
\begin{gathered}
\ldots, \mathrm{w}^{\prime}{ }_{2 i-1}\left(x_{\chi(\mathrm{i}+2)-1}\right), \mathrm{w}_{2 i-1}\left(x_{\chi(\mathrm{i}+2)-1}\right), 0,0, \ldots, \\
\ldots, 0,0, \mathrm{w}_{2 i-1}^{\prime}\left(x_{\chi(i)+1}\right), \mathrm{w}_{2 i-1}\left(x_{\chi(i)+1}\right), \ldots,
\end{gathered}
$$

and the next row (row with number $2 i$ ) differs from the mentioned one only by the fact that $w_{2 i-1}$ should be written instead of $w_{2 i-1}$ everywhere. The numbers of the columns in which these nonzero elements are located are as follows.

$$
\begin{gathered}
2 \chi(i)-1,2 \chi(i), 2 \chi(i)+1,2 \chi(i)+2, \ldots \\
\ldots, 2 \chi(\mathrm{i}+2)-5,2 \chi(\mathrm{i}+2)-4
\end{gathered}
$$

the total number of such columns is $2(\chi(i+2)-\chi(i))-2$.

If $i$ is replaced by $i+1$, then it is necessary to consider rows with numbers $j \in\{2 \mathrm{i}+1,2 \mathrm{i}+2\}$; the sets of their nonzero elements will shift so that their beginning will be in the column with the number $2 \chi(i+1)-1:$

$$
\begin{gathered}
2 \chi(\mathrm{i}+1)-1,2 \chi(\mathrm{i}+1), 2 \chi(\mathrm{i}+1)+1, \\
2 \chi(i+1)+2, \ldots, 2 \chi(\mathrm{i}+3)-5,2 \chi(\mathrm{i}+3)-4
\end{gathered}
$$

The numbers of the common columns in $\left(3.39^{*}\right)$ and $\left(3.39^{* *}\right)$ are as the follows

$$
\begin{gathered}
2 \chi(\mathrm{i}+1)-1,2 \chi(\mathrm{i}+1), 2 \chi(\mathrm{i}+1)+1 \\
2 \chi(i+1)+2, \ldots, 2 \chi(\mathrm{i}+2)-5,2 \chi(\mathrm{i}+2)-4 .
\end{gathered}
$$

Since the multiplicity of the covering by the supports of the coordinate functions $w_{j}$ is equal to four, the columns of this matrix contain no more than four nonzero elements (in consecutive four rows), and the matrix itself has an obvious stepped structure.

\section{References}

1. D. Černá. Postprocessing Galerkin method using quadratic spline wavelets and its efficiency. Comp. and Math. with App. 75(9) pp. 3186-3200 (2018)

2. J. Chen, W. Tang, M. Xu. Meshless analysis method of ship structures based on a B-spline wavelet. Harbin Gongcheng Daxue Xuebao/J. of Harbin Engineering Univ., 37(1) pp.13-18 (2016)

3. X. Zhang, R.X. Gao, R. Yan, X. Chen, C. Sun, Z. Yang. Analysis of laminated plates and shells using b-spline wavelet on interval finite element. International J. of Structural Stability and Dynamics, 17(6) (2017)

4. R. Boujelbene, Y. Ben Jemaa, M. Zribi. Toward an optimal B-spline wavelet transform for image compression. Proceedings of IEEE/ACS International Conference on Computer Systems and Applications, AICCSA, paper N 7945738 (2017)

5. C. Wang, Z.H. Gao, J.T. Huang, K. Zhao, J. Li. Smoothing methods based on coordinate transformation in a linear space and application in airfoil aerodynamic design optimization. Science China Technological Sciences, 58(2) pp297-306

6. Ö. Oruç. A numerical procedure based on Hermite wavelets for two-dimensional hyperbolic telegraph equation. Engineering with Comp. 34(4) pp741-755 (2018)

7. E. Ashpazzadeh, B. Han, M. Lakestani. Biorthogonal multiwavelets on the interval for numerical solutions of Burgers' equation. J. of Comp. and App. Math. 317 pp.510-534 (2017)

8. Z.M. Sulaimanov, B.M. Shumilov. A splitting algorithm for the wavelet transform of cubic splines on a nonuniform grid. Comp. Math. and Math. Phys. 57(10) pp.1577-1591 (2017)

9. Yu.K.Dem'yanovich. On embedding and extended smoothness of spline spaces. Far East J. of Math. Sciences (FJMS) 102, pp.2025-2052 (2017) 